\title{
Dielectric relaxation and piezoelectric coupling in the mixed proton-glass crystal $\mathrm{K}_{0.61}\left(\mathrm{NH}_{4}\right)_{0.39} \mathrm{H}_{2} \mathrm{PO}_{4}$
}

\author{
C.-S. Tu, V. Hugo Schmidt, and Adli A. Saleh \\ Department of Physics, Montana State University, Bozeman, Montana 59717
}

(Received 7 June 1993)

\begin{abstract}
Measurements of the dielectric permittivities in the mixed proton-glass crystal $\mathrm{K}_{0.61}\left(\mathrm{NH}_{4}\right)_{0.39} \mathrm{H}_{2} \mathrm{PO}_{4}$ are reported from $20 \mathrm{~Hz}$ to $200.8 \mathrm{MHz}$ and for fields along both the $a$ and $c$ axes. Between 14 and $50 \mathrm{~K}$, the $c$-axis permittivity outside the piezoelectric-resonance range shows a temperature-dependent spread in relaxation times but cannot be fit accurately with the Cole-Cole formula. Strong piezoelectric resonance for a field along the $c$ axis was observed in the region between $100 \mathrm{kHz}$ and $10 \mathrm{MHz}$.
\end{abstract}

\section{INTRODUCTION}

Among structurally frustrated mixed crystals, ${ }^{1}$ solid solutions of rubidium dihydrogen phosphate (RDP) and ammonium dihydrogen phosphate (ADP) have attracted considerable attention since 1982. In these systems, there is competition between the ferroelectric (FE) ordering of RDP and the antiferroelectric (AFE) ordering of ADP, each characterized by specific configurations of the acid protons. The random distribution of the $\mathrm{Rb}$ and $\mathrm{NH}_{4}$ ions in RADP produces frustration. However, little progress has been made in measuring glasslike properties of mixed crystals of $\mathrm{KDP}\left(\mathrm{KH}_{2} \mathrm{PO}_{4}\right)$ and ADP $\left(\mathrm{NH}_{4} \mathrm{H}_{2} \mathrm{PO}_{4}\right)$ since the discovery of dispersion phenomena in this system. ${ }^{2-6}$ The main reason is probably the difficulty of crystal growth. In this paper, we report our dielectric investigations and the temperature-dependent piezoelectric coupling effect $^{7,8}$ of the mixed crystal $\mathrm{K}_{1-x}\left(\mathrm{NH}_{4}\right)_{x} \mathrm{H}_{2} \mathrm{PO}_{4}(x=0.39)$ from dielectric measurements. In particular, the Curie-Weiss constant and elastic compliance (at $T=14.1 \mathrm{~K}$ ) were estimated by curve fitting.

Crystals of the KDP family are already piezoelectric in the paraelectric phase, so it is necessary to consider their dielectric and elastic responses simultaneously. Audio frequencies are below the sample piezoresonances, whereas microwave frequencies are above them. Hence, it is necessary to transform the "free" dielectric results to "clamped" values at audio frequencies to perform a comparison.

Dielectric measurements can provide important insight into the dynamics of molecular and ionic matter. For the complete characterization of the relaxation and resonant motional mechanisms involved, a large temperature range and a broad spectral frequency range are required. We report here an observation of frequency dispersion and piezoelectric resonance in the mixed crystal $\mathrm{K}_{0.61}\left(\mathrm{NH}_{4}\right)_{0.39} \mathrm{H}_{2} \mathrm{PO}_{4}$, made in the region between $20 \mathrm{~Hz}$ and $200.8 \mathrm{MHz}$.

\section{SAMPLE PREPARATION AND DETERMINATION OF CONCENTRATION}

Single mixed crystals of $(\mathrm{KDP})_{0.61}(\mathrm{ADP})_{0.39}$ were prepared by slow evaporation of a saturated aqueous solution with molar ratio $60: 40$ [KDP]:[ADP] at room temperature. The $c$ - and $a$-cut samples of dimensions $3.8 \times 2.8 \times 1.2 \mathrm{~mm}^{3}$ and $4.5 \times 3.7 \times 1.0 \mathrm{~mm}^{3}$, respectively, were electroded by silver evaporation on the polished sample surfaces.

Rutherford backscattering spectrometry was used to determine the composition of the mixed crystal. In this technique, a beam of helium or hydrogen ions with an energy of 1-2 MeV is incident on the sample. The basic principles of this technique are well known. ${ }^{9}$ If a sample consists of the elements $A_{1}, A_{2}, \ldots, A_{n}$ with the concentrations $x_{1}, x_{2}, \ldots, x_{n}$, respectively, the backscattering spectrum consists of $n$ steps each corresponding to an element. The height of the step due to element $A_{i}$ at its edge is proportional to the atomic density $N_{i}$ as well as the Rutherford collision cross section. The relative concentration was determined by comparing the two steps due to potassium and phosphorus after normalizing the height of each step to the corresponding scattering cross section.

We find that the relation between the ammonium concentration $x=0.39 \pm 0.01$ in the crystal and the composition $x_{s}=0.40 \pm 0.01$ in the aqueous solution from which the crystal was grown near $25^{\circ} \mathrm{C}$ differs considerably from previously reported $x$ vs $x_{s}$ plots. $^{2,3}$ Specifically, for $x_{s}=0.40$, Refs. 2 and 3 predict $x$ values of 0.12 for $20^{\circ} \mathrm{C}$ growth and 0.09 for $0{ }^{\circ} \mathrm{C}$ growth, respectively. This discrepancy cannot be resolved by assuming that our crystal actually had $x=0.12$ composition, because then we should have found a sharp $\epsilon_{c}^{\prime}$ peak near $85 \mathrm{~K}$ as found $^{2}$ for $x=0.11$ instead of our observed broad peak near $40 \mathrm{~K}$, which resembles results found ${ }^{5}$ for $x=0.32$. We have no explanation for this discrepancy.

\section{EXPERIMENT}

Two different measurement setups were used for the low- and high-frequency regions. For low frequencies, in the range between $20 \mathrm{~Hz}$ and $300 \mathrm{kHz}$, we used a Wayne-Kerr Model 6425 Precision Component Analyzer with four-lead connections and a closed-cycle refrigerator which can reach $10 \mathrm{~K}$. A silicon diode temperature sensor was put immediately below the sample holder. The measuring field strength was 5 volts $/ \mathrm{cm}$ and the samples were cooled at a rate of about $0.5 \mathrm{~K} / \mathrm{min}$. The errors in 
temperature measurement and the dielectric relative permittivities $\left(\varepsilon^{\prime}\right.$ and $\left.\varepsilon^{\prime \prime}\right)$ are about $\pm 0.1 \mathrm{~K}$ and $0.05 \%$, respectively.

For higher frequencies ( $1 \mathrm{MHz}$ to $1 \mathrm{GHz}$ ), the selfbalancing Hewlett-Packard 4191A RF Impedance Analyzer was used under computer control with the sample in a cryostat. The details of this experimental technique have been discussed elsewhere. ${ }^{10}$ In this work the DT-422 silicon diode temperature sensor was put right below the sample by drilling a hole through the ratchet. The accuracy of temperature measurement is about $\pm 0.01 \mathrm{~K}$. From the complex reflection coefficient, the relative permittivity $\varepsilon=\varepsilon^{\prime}-i \varepsilon^{\prime \prime}$ was determined at about 60 frequencies between $1 \mathrm{MHz}$ and $1 \mathrm{GHz}$. Between 10 and $60 \mathrm{~K}$, sharp piezoresonances were observed on the $c$ cut crystal at frequencies below $10 \mathrm{MHz}$. However, due to intrinsic circuit effects caused by the presence of residual parameters and stray capacitance (refer to pp. 3-48,49 of the HP4191A manual), the dielectric permittivity above $300 \mathrm{MHz}$ shows a frequency-dependent intrinsic circuit resonance. We have measured crystals of several different kinds and shapes (RADA-10, ADP, KADP-39, Teflon, and $\mathrm{Na}_{0.5} \mathrm{Bi}_{0.5} \mathrm{TiO}_{3}$, etc.). In each case, the frequency range yielding highly accurate results was limited to $300 \mathrm{MHz}$ in the high-frequency measuring system. We also tested Teflon cylinders with different thicknesses (all small compared to the diameter) at room and liquid- $N_{2}$ temperature. We found that the real part of the relative permittivity is 2.5 and independent of sample thickness and frequency over the whole range from 1 to $1000 \mathrm{MHz}$. The imaginary part of the relative permittivity is zero only below $300 \mathrm{MHz}$, and indicates slightly negative values, about $1 \%$ of the real part, above this frequency. Considering the variability of the plastics from different sources, the agreement is good below $300 \mathrm{MHz}$ with the quoted values ${ }^{11}$ of $\varepsilon^{\prime}=2.0$, and $\varepsilon^{\prime \prime}=0.0002$ both at $60 \mathrm{~Hz}$ and $1 \mathrm{MHz}$. We believe that this kind of limit is due to the instrumental intrinsic circuit effect caused by the presence of residual parameters and stray capacitance especially at frequencies above $300 \mathrm{MHz}{ }^{12}$ For these reasons, only results in the region between 21 and 200.8 $\mathrm{MHz}$ are presented for the relaxation behavior. All the data were collected automatically with the frequency swept in logarithmically spaced steps from $1 \mathrm{MHz}$ to 1 GHz. The errors of the relative permittivities $\left(\varepsilon^{\prime}\right.$ and $\left.\varepsilon^{\prime \prime}\right)$ are about $0.5 \%$.

\section{RESULTS AND DISCUSSION}

Plots of the real part of the $c$-axis relative permittivity $\varepsilon_{c}^{\prime}$ vs temperature at five typical frequencies are shown in Fig. 1(a). The low-frequency curves illustrate the onset of dispersion below $\sim 40 \mathrm{~K}$. The loss curves also exhibit dispersion, as shown in Fig. 1(b). Strong piezoelectric resonance occurs in the region between $100 \mathrm{kHz}$ and 10 MHz. Above this resonance, the sample is effectively clamped, as illustrated by the 100.9 and $200.8 \mathrm{MHz}$ curves. Below $\sim 50 \mathrm{~K}$, the high-frequency curves up to $200.8 \mathrm{MHz}$ show a small dispersion related to the distribution of relaxation times. The real part of the $a$-axis relative permittivity $\varepsilon_{a}^{\prime}$ was also measured as a function of temperature. Only a very small dispersion was observed, as shown in Fig. 1(c).

By fitting the $\varepsilon_{c}^{\prime}$ vs temperature curves with a value of $\varepsilon_{\infty}=10$, which is typical for crystals of this type, we find that the values $\varepsilon_{c}^{\prime}(T)$ at $1 \mathrm{kHz}$ for $T>T_{f} \sim 94 \mathrm{~K}$ follow a Curie-Weiss law, $\varepsilon_{c}^{\prime}-\varepsilon_{\infty}=C /\left(T-T_{0}\right)$, with $C=2776 \pm 1$ $\mathrm{K}$ and $T_{0}=59.1 \pm 0.1 \mathrm{~K}$. Here, $T_{f}(f)$ is the frequencydependent "freezing temperature" below which the polarization cannot follow the applied ac field, so that the $\varepsilon_{c}^{\prime}(f)$ curve can no longer follow the zero-frequency curve. Similarly, for $200.8 \mathrm{MHz}$, these parameters are $C=2896 \pm 1 \mathrm{~K}, T_{0}=57.3 \pm 0.1 \mathrm{~K}$, and $T_{f} \sim 96 \mathrm{~K}$. One notes that the clamped value of $T_{0}$ is lower than the Curie-Weiss temperature of the free sample. This shift between free and clamped Curie temperatures is much smaller than the value for RADP-35. ${ }^{13}$

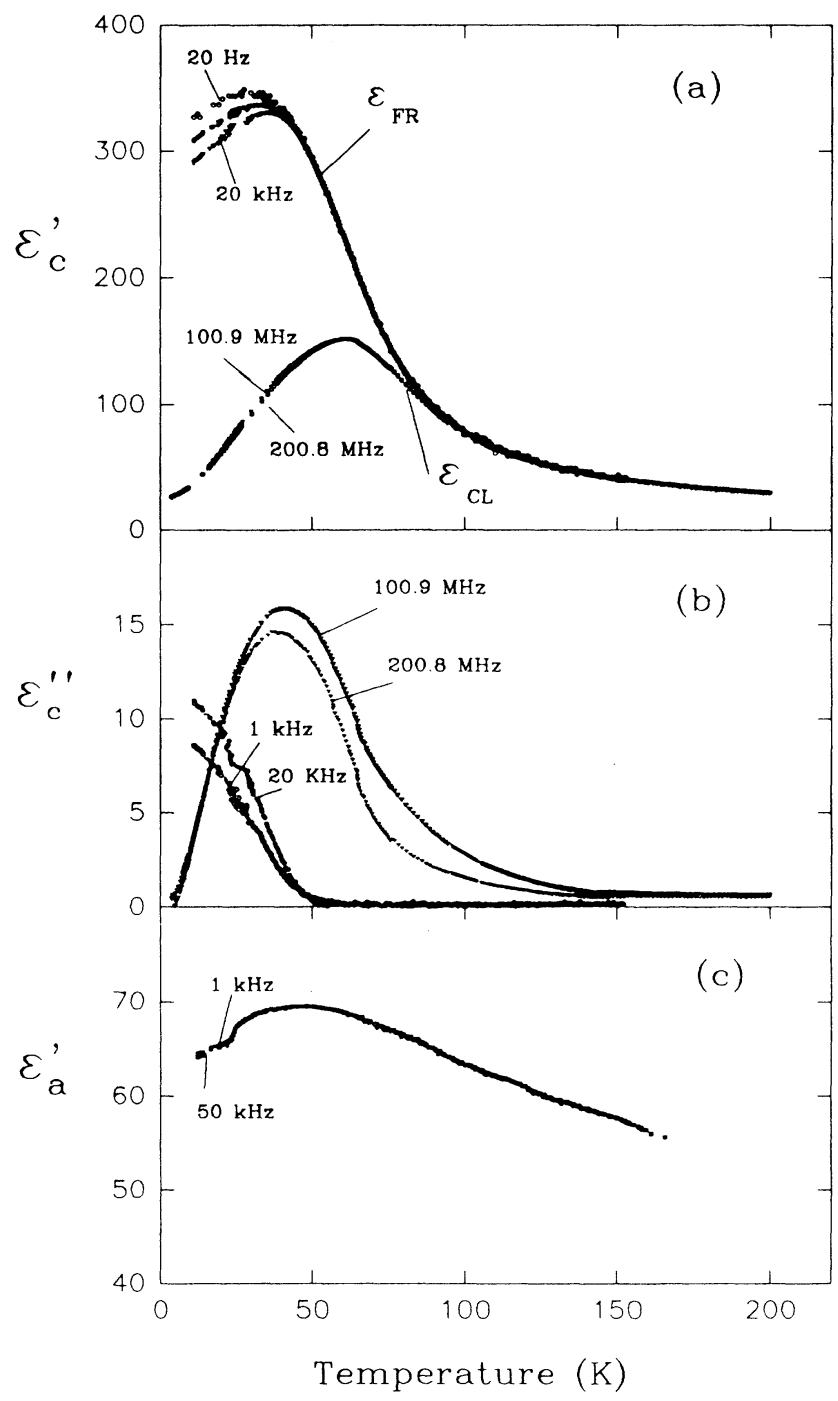

FIG. 1. (a) Dielectric permittivity $\varepsilon_{c}^{\prime}$ vs $T$ below the piezoelectric resonances (at $20 \mathrm{~Hz}, 1 \mathrm{kHz}$, and $20 \mathrm{kHz}$ ) and above them (at 100.9 and $200.8 \mathrm{MHz}$ ). Temperature dependence of (b) the dielectric loss $\varepsilon_{c}^{\prime \prime}$ and (c) the dielectric permittivity $\varepsilon_{a}^{\prime}$ at several frequencies. 
The piezoelectric coupling constant $Q$ can be determined from a measurement of fully relaxed free and clamped dielectric permittivities $\varepsilon_{\mathrm{FR}}$ and $\varepsilon_{\mathrm{CL}}$, respectively, since ${ }^{13}$

$$
Q^{-1}=1-\left(\varepsilon_{\mathrm{CL}}-1\right) /\left(\varepsilon_{\mathrm{FR}}-1\right) .
$$

Here, we use the dielectric responses at $20 \mathrm{~Hz}$ and 200.8 $\mathrm{MHz}$ for $\varepsilon_{\mathrm{FR}}$ and $\varepsilon_{\mathrm{CL}}$, respectively, to estimate the coupling constant $Q .{ }^{13}$ The result is displayed in Fig. 2 and our curve differs from that found ${ }^{13}$ for RADP-35 by starting lower and increasing much more rapidly as temperature increases. In order to make quantitative comparisons for dielectric permittivity, the free dielectric results, $\varepsilon^{f}(v, T)$ need to be transformed into the clamped values, using ${ }^{14}$

$$
\begin{aligned}
{\left[\varepsilon^{c}(\nu, T)-1\right]^{-1}=} & {\left[\varepsilon^{f}(v, T)-1\right]^{-1} } \\
& +Q^{-1}\left[\varepsilon^{c}(0, T)-1\right]^{-1} .
\end{aligned}
$$

The transformed data are presented in the form of $\varepsilon_{c}^{\prime \prime}$ vs $\varepsilon_{c}^{\prime}$ in Fig. 3. Apparently, the plot of $\varepsilon_{c}^{\prime \prime}$ vs $\varepsilon_{c}^{\prime}$ in the high-frequency region above $21 \mathrm{MHz}$ cannot be fit accurately with the Cole-Cole ${ }^{14}$ distribution, which gives a circular arc with depressed origin. The data fit somewhat better the Cole-Davidson ${ }^{14}$ distribution which gives a skewed arc. The data of Brückner, Courtens, and Unruh $^{15}$ for RADP-35 at high frequency fit a Cole-Cole distribution except at their lowest temperature where their plot was almost flat, resembling the left sides of the curves in Fig. 3.

The frequency vs dielectric permittivity $\left(f-\varepsilon_{c}^{\prime}\right)$ plots [Fig. 4(a)] at two different temperatures show the presence of piezoresonances occurring in the region between $100 \mathrm{kHz}$ and $10 \mathrm{MHz}$, with the resonance peak near 1 MHz. Figure 4 also shows that the resonance curve shapes are temperature dependent. The reason is that the elastic stiffness tensor, as well as the coupling tensors, are

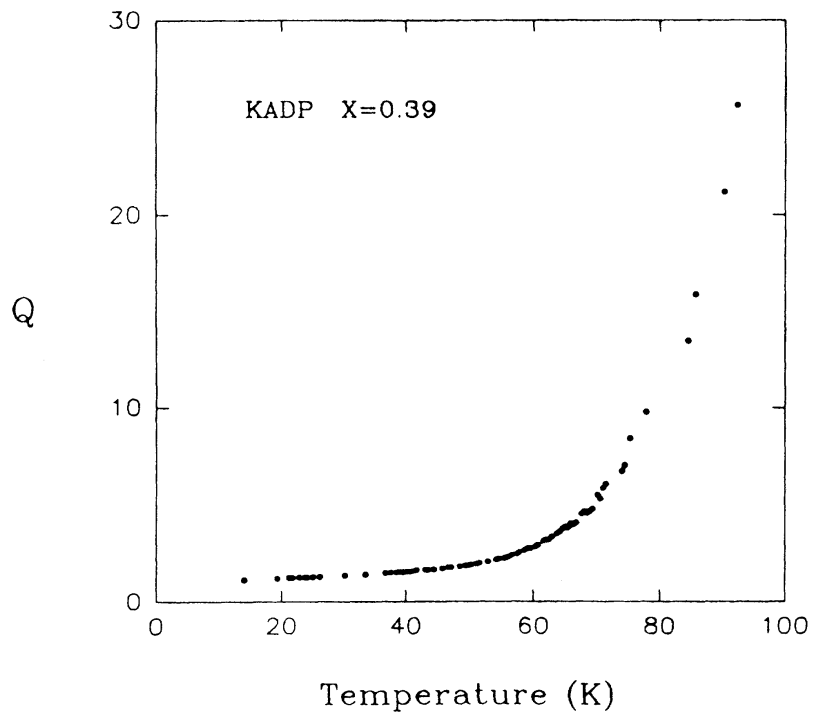

FIG. 2. Piezoelectric coupling parameter $Q$ derived from the dielectric results of Fig. 1(a).

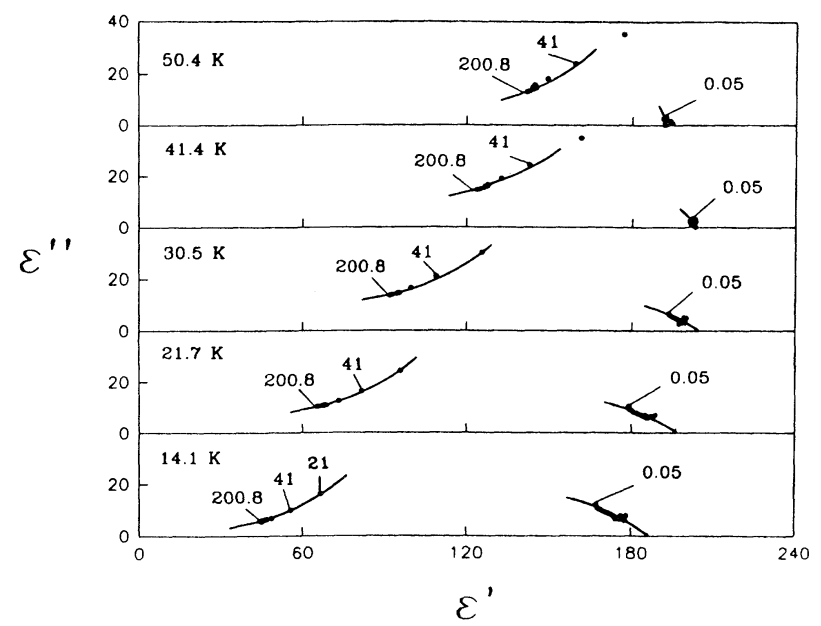

FIG. 3. Complex representation of the KADP-39 dielectric data at different temperatures. The numbers on the points indicate the frequencies in $\mathrm{MHz}$. The solid lines are guides to the eye.

dependent on temperature. The temperature dependence of the elastic stiffness originates from lattice anharmonicity. Apparently, in the region between $100 \mathrm{kHz}$ and 10 $\mathrm{MHz}$, it is difficult to distinguish the orientational relaxation of dipolar motion and the piezoresonance coupling effect.

Surprisingly, the dielectric constants $\varepsilon_{a}^{\prime}$ and $\varepsilon_{a}^{\prime \prime}$ shown in Fig. 5 also show a very small resonance effect around 1 MHz. We suspect that this is due to a slight misalignment of the field, and a small contribution of the $c$-axis piezoresonance, because the crystal symmetry forbids piezoelectricity for fields along the $a$ axis. The loss component $\varepsilon_{a}^{\prime \prime}$ in Fig. 5 is small, compared to the corresponding loss components in RADP and RADA, because the temperature is low and the real part [Fig. 1(c)] is rather small.

The inset in Fig. 6 is an equivalent circuit for a piezoelectric resonator in which the $C, R$, and $L$ components correspond, respectively, to the elasticity, viscosity, and mass of the sample. The solid line in Fig. 6 was plotted according to the real part of the resonant equation with $C^{x}=4, \quad C=16, \quad L=1.0 \times 10^{-14}$, and $R=6.0 \times 10^{-9}$ (in mks units). ${ }^{8}$ If the sample is in rectangular form, the resonance frequency and elastic constants for the longitudinal resonance mode can be estimated by using the following equations: ${ }^{8}$

$$
\begin{aligned}
& f_{0}=\left[2 \pi(L C)^{1 / 2}\right]^{-1}=(2 \xi)^{-1}\left(\rho^{E} S_{33}\right)^{-1 / 2}, \\
& R=\left(2 \pi f_{0} C Q_{1}\right)^{-1}, Q_{1}=\left(\tan \delta_{M}\right)^{-1} .
\end{aligned}
$$

In the above expressions, $f_{0}$ is the resonant frequency, $\zeta$ the length, $\rho$ the density of the sample, ${ }^{E} S_{33}$ is the elastic compliance under constant field, $Q_{1}$ is the mechanical quality factor, and $1 / Q_{1}$ is the mechanical loss tangent $\left(\tan \delta_{M}\right)$. The density $\rho$ of the sample is $\sim 1890 \pm 10$ $\left(\mathrm{kg} / \mathrm{m}^{3}\right)$ at room temperature. By using Eqs. (3) and (4) with the parameters chosen by fitting the curve in Fig. 6, and assuming that the density $\rho$ is temperature indepen- 
dent, we find that the resonance frequency $f_{0}$ is $\sim 0.4$ $\mathrm{MHz}, Q_{1}$ is $\sim 4.25$ (i.e., $\tan \delta \sim 0.24$ ), and ${ }^{E} S_{33}$ is $\sim 0.6 \times 10^{-10}\left(\mathrm{~m}^{2} / \mathrm{N}\right)$ at temperature $14.1 \mathrm{~K}$.

\section{CONCLUSIONS}

Our results obtained at frequencies from $20 \mathrm{~Hz}$ to $200.8 \mathrm{MHz}$ confirm the existence of a broad distribution of relaxation times in KADP-39 between 14 and $50 \mathrm{~K}$, as illustrated in Fig. 3. However, the Cole-Cole and ColeDavidson distributions ${ }^{14}$ cannot fit Fig. 3 accurately in the high-frequency range.

We find that the dielectric permittivity $\varepsilon_{c}^{\prime}$ and loss $\varepsilon_{c}^{\prime \prime}$ of KADP-39 are higher than for RADP-35 crystals in the glasslike region. One mechanism that can confidently be ruled out for both crystals is ammonium reorientation, because it does not result in net charge redistribution and

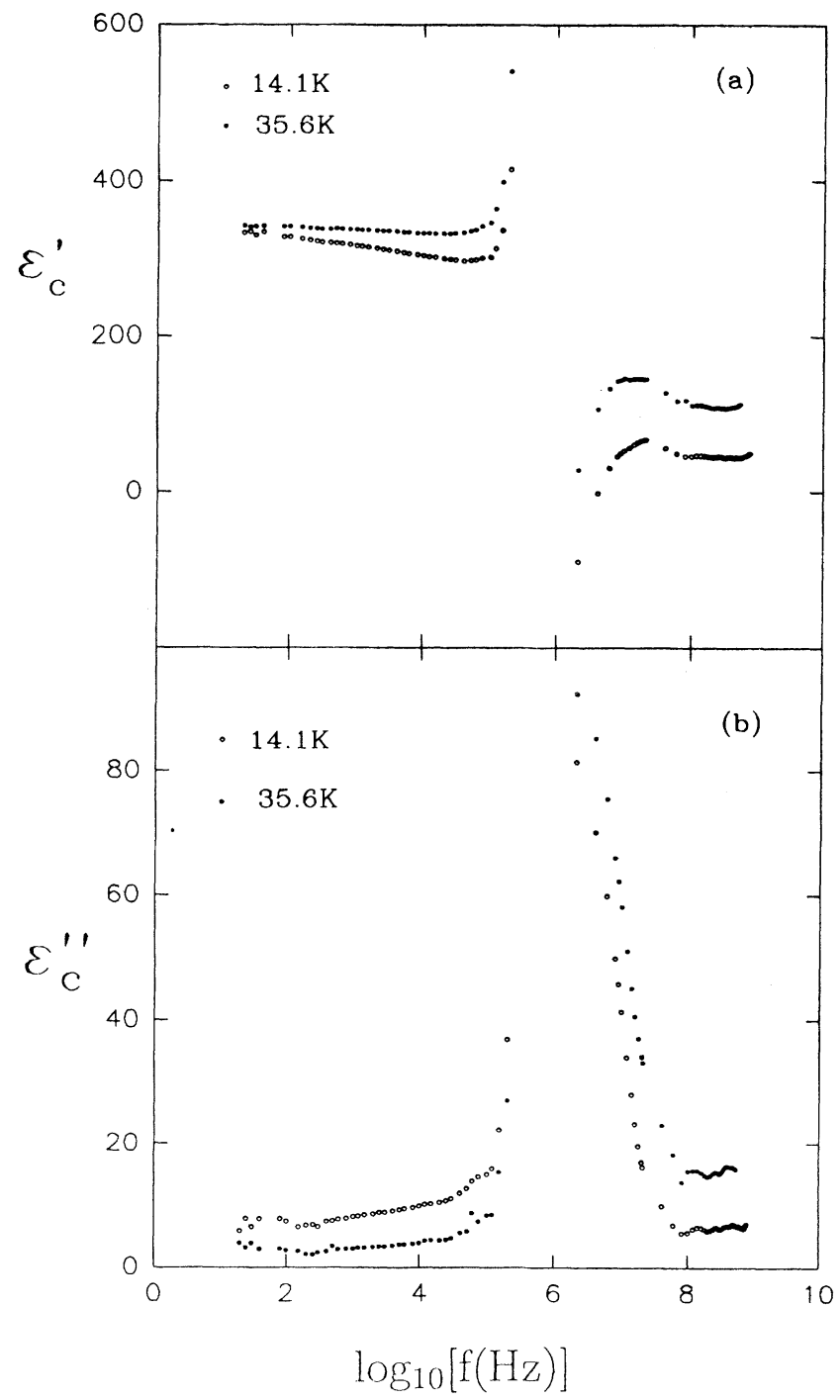

FIG. 4. Frequency dependence of (a) the dielectric permittivity $\varepsilon_{c}^{\prime}$ and (b) the dielectric loss $\varepsilon_{c}^{\prime \prime}$ at temperatures of 14.1 and $35.6 \mathrm{~K}$. because this motion is frozen out at low temperatures, as is known from NMR measurements. ${ }^{16}$ However, displacements of the entire ammonium ion can contribute, as can potassium and phosphorus ion displacements. If the KADP structure is looser than the RADP structure, these displacements could be larger, giving larger $\varepsilon_{c}^{\prime}$. The acid protons move almost perpendicular to the $c$ axis and cannot contribute directly to $\varepsilon_{c}^{\prime}$, but their motions trigger displacements of the heavier ions. Another feature of KADP-39, not seen in RADP-35 (Ref. 15) or even in KADP-32 (Ref. 5), is the persistence of a large $\varepsilon_{c}^{\prime \prime}$ to very low temperature. In this regard one should recall that the acid-proton motions are attributed both to thermally activated hopping and tunneling, so the persistence of these losses to very low temperature could be related to proton tunneling. ${ }^{17-19}$

From our measurements and fitting the data for our

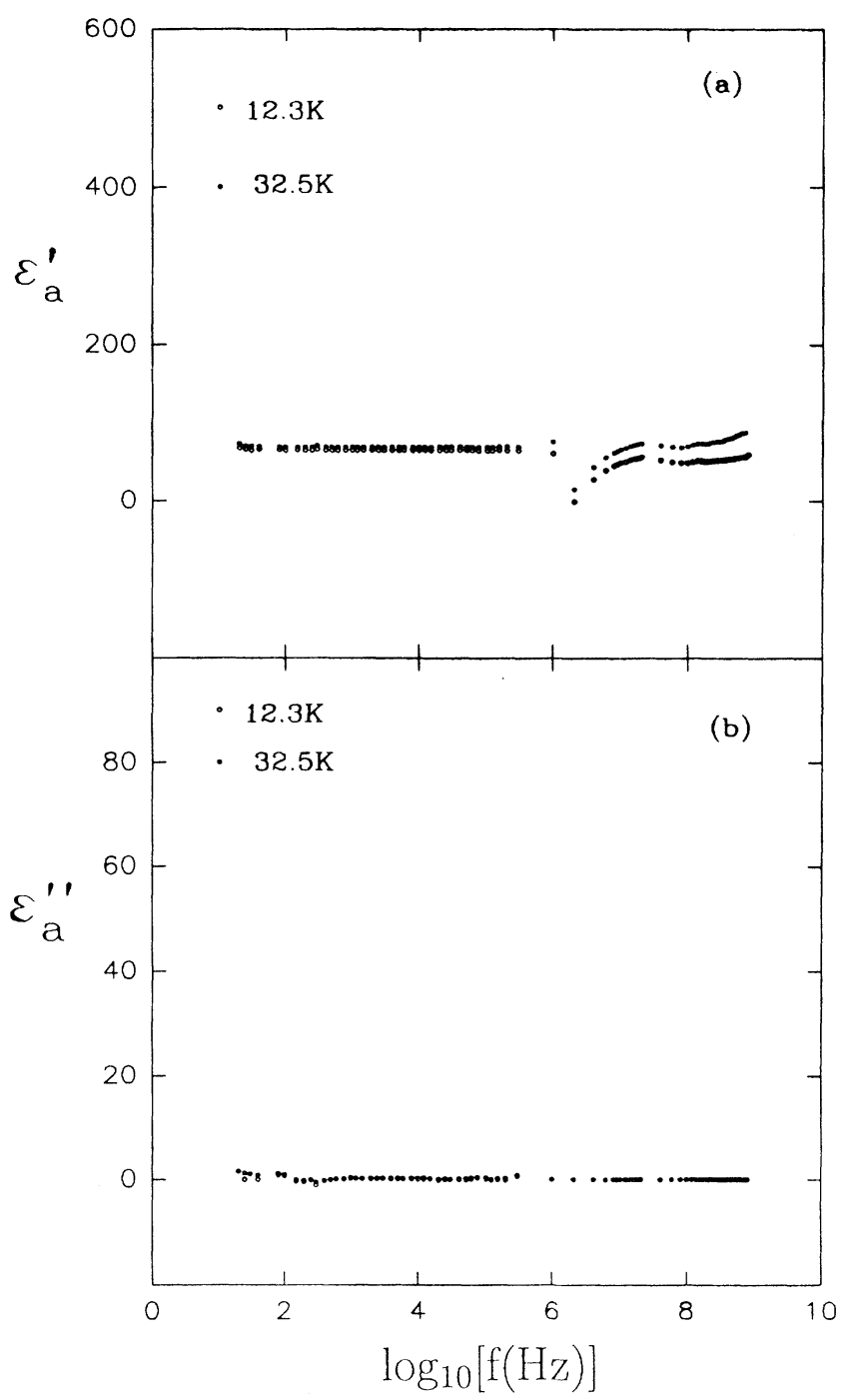

FIG. 5. Frequency dependence of (a) the dielectric permittivity $\varepsilon_{a}^{\prime}$ and (b) the dielectric loss $\varepsilon_{a}^{\prime \prime}$ at temperatures of 12.3 and $32.5 \mathrm{~K}$. 


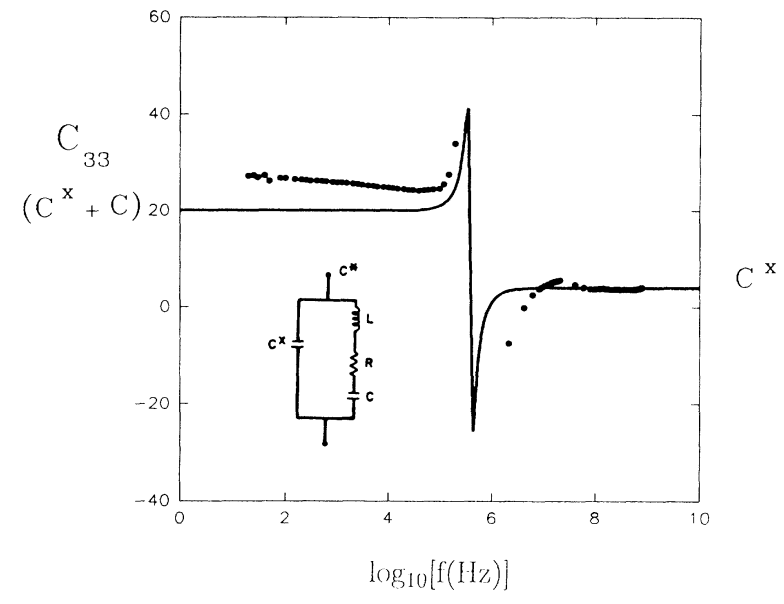

FIG. 6. Frequency dependence of the capacitance $C_{33}$ at temperature $14.1 \mathrm{~K}$. The solid line represents a fit to the real part of the complex capacitance with $C^{x}=4, C=16$, $L=1.0 \times 10^{-14}$, and $R=6.0 \times 10^{-9}$ (in mks units). $C^{x}$ is the capacitance of the sample without piezoelectric coupling.

$\mathrm{K}_{0.61}\left(\mathrm{NH}_{4}\right)_{0.39} \mathrm{H}_{2} \mathrm{PO}_{4}$ crystal, we find elastic coupling and dielectric dispersion similar to those found in the mixed crystal KADP-25, which showed a glassy dispersion and strong elastic coupling effect. ${ }^{2}$ In other words, crystals in the intermediate concentration region $(0.17 \leq x \leq 0.85)$ of KADP seem to be dominated by frustration which is caused by the structural competition between the FE and AFE ordering tendencies, and by piezoelectric coupling.

The exact mechanism of the glassy behavior in proton-glass crystals still is not well understood. In particular, the theory of KADP mixed crystals is as yet incomplete because both the $a$ - and $c$-axis permittivities differ considerably from those of RADP and RADA. We feel that further wide frequency-range studies of the type presented here are needed in the glasslike region of KADP.

\section{ACKNOWLEDGMENTS}

The authors would like to express sincere thanks to Norman Williams for his assistance in making apparatus, to Dr. Mario Maglione for a helpful discussion regarding the high-frequency bridge, to Dr. Stuart L. Hutton for his help in automating the measuring apparatus and providing ideas for setting up the high-frequency system, and to Professor George F. Tuthill for helpful discussions. This work was supported by National Science Foundation Grant No. DMR-9017429. The radio frequency impedance analyzer was purchased from funds provided by Department of Energy Equipment Grant No. DOEFG05-91ER79046.
${ }^{1}$ E. Courtens, Helv. Phys. Acta 56, 705 (1983).

${ }^{2}$ Y. Ono, T. Hikita, and T. Ikeda, J. Phys. Soc. Jpn. 56, 577 (1987).

${ }^{3}$ P. Askenasy and F. Nessler, Z. Anorg. Chem. 189, 305 (1930).

${ }^{4}$ Y. Ono, T. Hikita, and T. Ikeda, Ferroelectrics 79, 327 (1988).

${ }^{5}$ S. A. Gridnev, L. N. Korotkov, S. P. Rogova, L. A. Shuvalov, and R. M. Fedosjuk, Ferroelectrics Lett. 13, 67 (1991).

${ }^{6}$ A. I. Baranov, and V. H. Schmidt, in Proceedings of the 7th International Symposium on Applied Ferroelectrics (IEEE, Piscataway, 1991), p. 502.

${ }^{7}$ T. T. Wang, J. M. Herbert, and A. M. Glass, The Application of Ferroelectric Polymers (Blackie, New York, 1988), p. 98.

${ }^{8}$ H. Ohigashi, J. Appl. Phys. 47, 949 (1976).

${ }^{9}$ W. K. Chu, M.-A. Nicolet, and J. W. Mayer, Backscattering Spectrometry (Academic, New York, 1977).

${ }^{10}$ R. Böhmer, M. Maglione, P. Lunkenheimer, and A. Loidl, J.
Appl. Phys. 65, 901 (1989).

${ }^{11}$ Handbook of Chemistry and Physics, 51st ed. (Chemical Rubber, Cleveland, 1970), p. C-769.

${ }^{12}$ M. Maglione, R. Böhmer, A. Loidl, and U. T. Höchli, Phys. Rev. B 40, 11441 (1989).

${ }^{13}$ E. Courtens, R. Vacher, and Y. Dagorn, Phys. Rev. B 33, 7625 (1986).

${ }^{14}$ V. V. Daniel, Dielectric Relaxation (Academic, London, 1967).

${ }^{15}$ H. J. Brückner, E. Courtens, and H.-G. Unruh, Z. Phys. B 7, 337 (1988).

16J. Slak, R. Kind, R. Blinc, E. Courtens, and S. Zumer, Phys. Rev. B 30, 85 (1984).

${ }^{17}$ R. Blinc, J. Phys. Chem. Solids 13, 204 (1960).

${ }^{18}$ R. Pirc, B. Tadic, and R. Blinc, Z. Phys. B 61, 69 (1985).

${ }^{19}$ B. Tadic, R. Pirc, and R. Blinc, Z. Phys. B 75, 249 (1989). 